\title{
MULTILEVEL SYMMETRIZED TOEPLITZ STRUCTURES AND SPECTRAL DISTRIBUTION RESULTS FOR THE RELATED MATRIX SEQUENCES*
}

\author{
PAOLA FERRARI ${ }^{\dagger}$, ISABELLA FURCI ${ }^{\ddagger}$, AND STEFANO SERRA-CAPIZZANO $\$$,
}

\begin{abstract}
In recent years, motivated by computational purposes, the singular value and spectral features of the symmetrization of Toeplitz matrices generated by a Lebesgue integrable function have been studied. Indeed, under the assumptions that $f$ belongs to $L^{1}([-\pi, \pi])$ and it has real Fourier coefficients, the spectral and singular value distribution of the matrixsequence $\left\{Y_{n} T_{n}[f]\right\}_{n}$ has been identified, where $n$ is the matrix size, $Y_{n}$ is the anti-identity matrix, and $T_{n}[f]$ is the Toeplitz matrix generated by $f$. In this note, the authors consider the multilevel Toeplitz matrix $T_{\mathbf{n}}[f]$ generated by $f \in L^{1}\left([-\pi, \pi]^{k}\right), \mathbf{n}$ being a multi-index identifying the matrix-size, and they prove spectral and singular value distribution results for the matrixsequence $\left\{Y_{\mathbf{n}} T_{\mathbf{n}}[f]\right\}_{\mathbf{n}}$ with $Y_{\mathbf{n}}$ being the corresponding tensorization of the anti-identity matrix.
\end{abstract}

Key words. Toeplitz matrices, Hankel matrices, Symmetrization, Singular value distribution, Eigenvalue distribution.

AMS subject classifications. 15B05, 47B06, 65F15, 15A18.

1. Introduction. Spectral and singular value distribution results $[1,4,10,7,8,23,27,28]$ of structured matrix-sequences represent one among the key ingredients in the design and in the convergence analysis of several well-known (preconditioned) iterative methods $[18,11]$. In many contexts, symmetry is a particularly desirable property for a matrix when we want to solve an associated linear system with iterative methods. Hence, from the original work by Pestana and Wathen [17], symmetrization procedures combined with various preconditioning techniques have been introduced and studied for the very purpose of developing a competitive method for the solution of real nonsymmetric structured systems.

Here it is worth mentioning that the notion of symmetrization is in a specific sense of equivalent linear systems and in fact the original coefficient matrix is multiplied from the left by an invertible matrix which transforms the original nonsymmetric Toeplitz coefficient matrix into a symmetric Hankel matrix (see below and [17] for the original proposal in the unilevel Toeplitz setting).

In particular, the singular value and spectral features of the symmetrization of Toeplitz matrices generated by a Lebesgue integrable function have been recently discussed and exploited in several settings. Indeed, under the assumptions that $f$ belongs to $L^{1}([-\pi, \pi])$ and it has real Fourier coefficients, the spectral and singular value distribution of the matrix-sequence $\left\{Y_{n} T_{n}[f]\right\}_{n}$ has been studied, where $Y_{n}$ is the anti-identity matrix and $T_{n}[f]$ is the Toeplitz matrix generated by $f[5,12]$. Several extensions of the latter result have been also treated. For example, the generalization in the context of block structures is treated in [5], i.e. assuming $f$ a matrix-valued function, while the spectral distribution of matrix-sequences of the form $\left\{h\left(T_{n}[f]\right)\right\}_{n}$, with $h$ being an analytic function, is studied in [6].

\footnotetext{
*Received by the editors on November 21, 2020. Accepted for publication on April 21, 2021. Handling Editor: Dario Bini. Corresponding Author: Isabella Furci.

${ }^{\dagger}$ Department of Science and High Technology, University of Insubria, Como, 22100, Italy (pferrari@uninsubria.it). Supported by INdAM Research group GNCS.

$\ddagger$ Department of Mathematics and Informatics, University of Wuppertal, Wuppertal, 42119, Germany (furci@uniwuppertal.de). Supported by INdAM Research group GNCS.

$\S$ Department of Humanities and Innovation, University of Insubria, Como, 22100, Italy (s.serracapizzano@uninsubria.it). Supported by INdAM Research group GNCS.

^Department of Information Technology, Uppsala University, SE-751 05 Uppsala, SWEDEN (stefano.serra@it.uu.se).
} 
The purpose of this paper is to extend the result concerning the eigenvalue and singular value distributions of the unilevel matrix-sequence $\left\{Y_{n} T_{n}[f]\right\}_{n}$ to the symmetrization of multilevel matrix-sequences of the form $\left\{T_{\mathbf{n}}[f]\right\}_{\mathbf{n}}$, where $f$ is a $k$-variate function $f \in L^{1}\left([-\pi, \pi]^{k}\right)$. The proof of the main Theorem of the paper is based on the relation between a Toeplitz matrix and its generating function $f$ and on the notion of approximating class of sequences (a.c.s.), as it has been done in the simpler unilevel case in [5]. Hence, the proof technique is not new. However, the multilevel adaptations are never obvious, and the multilevel setting is always worth of a clear presentation. Furthermore, it should be noted that the approach and the proof techniques in [12] and in the very recent report [13] are different since the authors employ the powerful Generalized Locally Toeplitz (GLT) matrix-sequences technology, which in turn heavily relies on the a.c.s. notion. Consequently, our proof is more elementary since it does not require the knowledge of the GLT machinery and the final result in terms of distribution is equivalent but simpler. In fact, the distribution function is not unique, while the set of possible distribution functions form an equivalence class under rearrangements: for the matter of comparison, we emphasize that our distribution function is scalar-valued versus the one in [13] which is $2 \times 2$ matrix-valued.

These preliminary concepts concerning spectral distribution, singular value distribution, and the notion of a.c.s. are introduced in Section 2 in the general $k$-level setting. In Section 3, we give our main result on the asymptotic distributions of $\left\{Y_{\mathbf{n}} T_{\mathbf{n}}[f]\right\}_{\mathbf{n}}$, first considering the case where $f$ is a trigonometric polynomial, then extending the result to $f \in L^{1}\left([-\pi, \pi]^{k}\right), k>1$. In addition, Section 4 is devoted to further results and observations, where we dedicate particular attention to the case where $f$ is a $k$-variate separable function. In Section 5, we collect relevant experiments, showing the numerical validity and accuracy of our theoretical findings. Finally, Section 6 is devoted to conclusions and open problems.

2. Preliminaries on Toeplitz matrices. Here we define the multi-index $\mathbf{n}=\left(n_{1}, n_{2}, \ldots, n_{k}\right)$ where each $n_{j}$ is a positive integer. When writing the expression $\mathbf{n} \rightarrow \infty$, we mean that every component of the vector $\mathbf{n}$ tends to infinity, i.e. $\min _{1 \leq j \leq k} n_{j} \rightarrow \infty$. Furthermore, in the current multilevel context, it is convenient to use the Kronecker tensor product $\otimes$ for matrices, where $A \otimes B$ denotes the block matrix of the form $\left(a_{i, j} B\right)$ with $A=\left(a_{i, j}\right)$. In a function setting, writing $f=f_{1} \otimes f_{2}$ indicates a basic separable function $f(x, y)=f_{1}(x) f_{2}(y)$ where $x$ lies in the domain of $f_{1}$ and $y$ lies in the domain of $f_{2}$.

Let $f:[-\pi, \pi]^{k} \rightarrow \mathbb{C}$ be a function belonging to $L^{1}\left([-\pi, \pi]^{k}\right)$ and periodically extended to $\mathbb{R}^{k}$. We define $T_{\mathbf{n}}[f]$ the multilevel Toeplitz matrix of dimensions $N(\mathbf{n}) \times N(\mathbf{n})$, with $N(\mathbf{n})=n_{1} n_{2} \ldots n_{k}$, as follows

$$
T_{\mathbf{n}}[f]=\sum_{\left|j_{1}\right|<n_{1}} \cdots \sum_{\left|j_{k}\right|<n_{k}}\left(J_{n_{1}}^{j_{1}} \otimes \cdots \otimes J_{n_{k}}^{j_{k}}\right) \hat{f}_{\mathbf{j}}, \quad \mathbf{j}=\left(j_{1}, j_{2}, \ldots, j_{k}\right) \in \mathbb{Z}^{k}
$$

In the latter, the quantities

$$
\hat{f}_{\mathbf{j}}=\frac{1}{(2 \pi)^{k}} \int_{[-\pi, \pi]^{k}} f(\boldsymbol{\theta}) \mathrm{e}^{\iota\langle\mathbf{j}, \boldsymbol{\theta}\rangle} \mathrm{d} \boldsymbol{\theta}
$$

with $\langle\mathbf{j}, \boldsymbol{\theta}\rangle=\sum_{t=1}^{k} j_{t} \theta_{t}, \iota^{2}=-1$, are the Fourier coefficients of $f$ and $J_{n}^{j}$ is the $n \times n$ matrix whose $(l, h)$-th entry equals 1 if $(l-h)=j$ and 0 otherwise.

Lemma 2.1 ([8]). Let $f_{1}, \ldots, f_{k} \in L^{1}([-\pi, \pi]), \mathbf{n}=\left(n_{1}, n_{2}, \ldots, n_{k}\right) \in \mathbb{N}^{k}$. Then,

$$
T_{n_{1}}\left[f_{1}\right] \otimes \cdots \otimes T_{n_{k}}\left[f_{k}\right]=T_{\mathbf{n}}\left[f_{1} \otimes \cdots \otimes f_{k}\right]
$$


where the Fourier coefficients of $f_{1} \otimes \cdots \otimes f_{k}$ are given by

$$
\left(f_{1} \otimes \cdots \otimes f_{k}\right)_{\mathbf{j}}=\left(f_{1}\right)_{j_{1}} \ldots\left(f_{k}\right)_{j_{k}}, \quad \mathbf{j} \in \mathbb{Z}^{k}
$$

Throughout the paper, we indicate by $\left\{T_{\mathbf{n}}[f]\right\}_{\mathbf{n}}$ the matrix-sequence whose elements are the matrices $T_{\mathbf{n}}[f]$. The function $f$ is called the generating function of $T_{\mathbf{n}}[f]$.

If $f$ is complex-valued, then $T_{\mathbf{n}}[f]$ is non-Hermitian for all sufficiently large $\mathbf{n}$. Conversely, if $f$ is realvalued, then $T_{\mathbf{n}}[f]$ is Hermitian for all $\mathbf{n}$. If $f$ is real-valued and nonnegative, but not identically zero almost everywhere, then $T_{\mathbf{n}}[f]$ is Hermitian positive definite for all $\mathbf{n}$. If $f$ is real-valued and even, $T_{\mathbf{n}}[f]$ is symmetric for all $\mathbf{n}[14,3]$.

The singular value and spectral distribution of Toeplitz matrix-sequences have been well studied in the past few decades. Ever since Szegö in [10] showed that the eigenvalues of the Toeplitz matrix $T_{n}[f]$ generated by real-valued $f \in L^{\infty}([-\pi, \pi])$ are asymptotically distributed as $f$. Moreover, Avram and Parter $[1,15]$ proved that the singular values of $T_{n}[f]$ are distributed as $|f|$ for a complex-valued $f \in L^{\infty}([-\pi, \pi])$. Tyrtyshnikov and Zamarashkin [27, 25, 28] and, independently, Tilli [23] later extended the spectral and singular value theorems to Toeplitz matrices $T_{n}[f]$ generated by functions $f \in L^{1}([-\pi, \pi])$. Recently, Garoni, Serra-Capizzano, and Vassalos [9] provided the same theorem in the unilevel case based on the theory of Generalized Locally Toeplitz (GLT) sequences [7]. As for the changes in the singular value and spectral distribution of Toeplitz matrix-sequences after certain matrix operations that are related to our concerned problems, much work was done by Tyrtyshnikov and Serra-Capizzano in [26, 19, 20, 21].

All these consideration suggest that the candidate function that describes the asymptotic distributions of Toeplitz matrix-sequences is the generating function, but this is actually verified only under specific hypotheses. Indeed, if a Toeplitz matrix is not Hermitian, in general, it is quite intricate to describe its spectral properties studying the generating function: there are beautiful results by Tilli [24], but they hold when the range of the generating function satisfies very specific topological properties. However, the knowledge of the spectral and singular value information is crucial in the design proper and fast methods for the solution of Toeplitz systems. Then, the study of strategies and their properties that permit us to symmetrize Toeplitz linear systems is fundamental and convenient.

Throughout this work, we assume that $f \in L^{1}\left([-\pi, \pi]^{k}\right)$ and is periodically extended to $\mathbb{R}^{k}$. Furthermore, we follow all standard notation and terminology introduced in [7]: let $C_{c}(\mathbb{C})$ (or $C_{c}(\mathbb{R})$ ) be the space of complex-valued continuous functions defined on $\mathbb{C}$ (or $\mathbb{R}$ ) with bounded support and let $\eta$ be a functional, i.e. any function defined on some vector space which takes values in $\mathbb{C}$. Also, if $g: D \subset \mathbb{R}^{k} \rightarrow \mathbb{K}(\mathbb{R}$ or $\mathbb{C})$ is a measurable function defined on a set $D$ with $0<\mu_{k}(D)<\infty$, the functional $\eta_{g}$ is denoted such that

$$
\eta_{g}: C_{c}(\mathbb{K}) \rightarrow \mathbb{C} \text { and } \eta_{g}(F)=\frac{1}{\mu_{k}(D)} \int_{D} F(g(\mathbf{x})) \mathrm{d} \mathbf{x} .
$$

Definition 2.2 ([7, Definition 3.1] (Singular value and eigenvalue distribution of a matrix-sequence)). Let $\left\{A_{\mathbf{n}}\right\}_{n}$ be a matrix-sequence.

1. We say that $\left\{A_{\mathbf{n}}\right\}_{\mathbf{n}}$ has an asymptotic singular value distribution described by a functional $\eta$ : $C_{c}(\mathbb{R}) \rightarrow \mathbb{C}$, and we write $\left\{A_{\mathbf{n}}\right\}_{\mathbf{n}} \sim_{\sigma} \eta$, if

$$
\lim _{\mathbf{n} \rightarrow \infty} \frac{1}{N(\mathbf{n})} \sum_{j=1}^{N(\mathbf{n})} F\left(\sigma_{j}\left(A_{\mathbf{n}}\right)\right)=\eta(F), \quad \forall F \in C_{c}(\mathbb{R}) .
$$


If $\eta=\eta_{|f|}$ for some measurable $f: D \subset \mathbb{R}^{k} \rightarrow \mathbb{C}$ defined on a set $D$ with $0<\mu_{k}(D)<\infty$, we say that $\left\{A_{\mathbf{n}}\right\}_{\mathbf{n}}$ has an asymptotic singular value distribution described by $f$ and we write $\left\{A_{\mathbf{n}}\right\}_{\mathbf{n}} \sim_{\sigma} f$.

2. We say that $\left\{A_{\mathbf{n}}\right\}_{\mathbf{n}}$ has an asymptotic eigenvalue (or spectral) distribution described by a functional $\eta: C_{c}(\mathbb{R}) \rightarrow \mathbb{C}$, and we write $\left\{A_{\mathbf{n}}\right\}_{\mathbf{n}} \sim_{\lambda} \eta$, if

$$
\lim _{\mathbf{n} \rightarrow \infty} \frac{1}{N(\mathbf{n})} \sum_{j=1}^{N(\mathbf{n})} F\left(\lambda_{j}\left(A_{\mathbf{n}}\right)\right)=\eta(F), \quad \forall F \in C_{c}(\mathbb{C}) .
$$

If $\eta=\eta_{f}$ for some measurable $f: D \subset \mathbb{R}^{k} \rightarrow \mathbb{C}$ defined on a set $D$ with $0<\mu_{k}(D)<\infty$, we say that $\left\{A_{\mathbf{n}}\right\}_{\mathbf{n}}$ has an asymptotic eigenvalue (or spectral) distribution described by $f$ and we write $\left\{A_{\mathbf{n}}\right\}_{\mathbf{n}} \sim_{\lambda} f$.

In the following, the generalized Szegö theorem that describes the singular value and spectral distribution of Toeplitz sequences is given in the multivariate setting. We refer to [8, Theorem 3.5] for a proof that is based on the notion of approximating class of sequences given in Definition 2.4.

TheOREM 2.3. Let $f \in L^{1}\left([-\pi, \pi]^{k}\right)$, with $k \geq 1$. Then,

$$
\left\{T_{\mathbf{n}}[f]\right\}_{\mathbf{n}} \sim_{\sigma} f .
$$

Moreover if $f$ is a real-valued function, then

$$
\left\{T_{\mathbf{n}}[f]\right\}_{\mathbf{n}} \sim_{\lambda} f .
$$

Moreover, we introduce the following definitions and a key lemma in order to prove our main distribution results in the next section. Regarding the employed norms we use the following notation: $\|\cdot\|$ denotes the spectral norm for matrices (that is the maximal singular value, also called Schatten- $\infty$ norm), $\|\cdot\|_{1}$ denotes the trace norm for matrices (that is the sum of all the singular values, also called Schatten-1 norm), and $\|\cdot\|_{L^{1}}$ denotes the standard $L^{1}$ norm for functions.

Definition 2.4 ([8, Definition 2.6](Approximating class of sequences)). Let $\left\{A_{\mathbf{n}}\right\}_{\mathbf{n}}$ be a matrix-sequence and let $\left\{\left\{B_{\mathbf{n}, m}\right\}_{\mathbf{n}}\right\}_{m}$ be a sequence of matrix-sequences. We say that $\left\{\left\{B_{\mathbf{n}, m}\right\}_{\mathbf{n}}\right\}_{m}$ is an approximating class of sequences (a.c.s) for $\left\{A_{\mathbf{n}}\right\}_{\mathbf{n}}$ if the following condition is met: for every $m$ there exists $n_{m}$ such that, for $n \geq n_{m}$,

$$
A_{\mathbf{n}}=B_{\mathbf{n}, m}+R_{\mathbf{n}, m}+N_{\mathbf{n}, m}
$$$$
\operatorname{rank} R_{\mathbf{n}, m} \leq c(m) N(\mathbf{n}) \quad \text { and } \quad\left\|N_{\mathbf{n}, m}\right\| \leq \omega(m),
$$

where $n_{m}, c(m)$, and $\omega(m)$ depend only on $m$ and

$$
\lim _{m \rightarrow \infty} c(m)=\lim _{m \rightarrow \infty} \omega(m)=0 .
$$

We use $\left\{B_{\mathbf{n}, m}\right\}_{\mathbf{n}} \stackrel{\text { a.c.s. wrt } m}{\longrightarrow}\left\{A_{\mathbf{n}}\right\}_{\mathbf{n}}$ to denote that $\left\{\left\{B_{\mathbf{n}, m}\right\}_{\mathbf{n}}\right\}_{m}$ is an a.c.s for $\left\{A_{\mathbf{n}}\right\}_{\mathbf{n}}$.

The following is a useful criterion to identify an a.c.s. without constructing the splitting present in Definition 2.4 .

THEOREM 2.5. Let $\left\{A_{\mathbf{n}}\right\}_{\mathbf{n}}$ be a sequence of matrices, with $A_{\mathbf{n}}$ of size $N(\mathbf{n})$, let $\left\{\left\{B_{\mathbf{n}, m}\right\}_{\mathbf{n}}\right\}_{m}$ be a sequence of matrix-sequences, with $B_{\mathbf{n}, m}$ of size $N(\mathbf{n})$. Suppose that for every $m$ there exists $\mathbf{n}_{m}$ such that, for $\mathbf{n} \geq \mathbf{n}_{m}$

$$
\left\|A_{\mathbf{n}}-B_{\mathbf{n}, m}\right\|_{1} \leq \epsilon(m) N(\mathbf{n})
$$


where $\lim _{m \rightarrow \infty} \epsilon(m)=0$. Then,

$$
\left\{B_{\mathbf{n}, m}\right\}_{\mathbf{n}} \stackrel{\text { a.c.s. wrt } m}{\longrightarrow}\left\{A_{\mathbf{n}}\right\}_{\mathbf{n}} .
$$

Lemma 2.6 ([8, Corollary 2.4]). Let $\left\{A_{\mathbf{n}}\right\}_{\mathbf{n}},\left\{B_{\mathbf{n}, m}\right\}_{\mathbf{n}}$ be matrix-sequences and let $f, f_{m}: D \subset \mathbb{R}^{k} \rightarrow \mathbb{C}$ be measurable functions defined on a set $D$ with $0<\mu_{k}(D)<\infty$. Suppose that

1. $\left\{B_{\mathbf{n}, m}\right\}_{\mathbf{n}} \sim_{\sigma} f_{m}$ for every $m$,

2. $\left\{B_{\mathbf{n}, m}\right\}_{\mathbf{n}} \stackrel{\text { a.c.s. wrt } m}{\longrightarrow}\left\{A_{\mathbf{n}}\right\}_{\mathbf{n}}$,

3. $f_{m} \rightarrow f$ in measure.

Then

$$
\left\{A_{\mathbf{n}}\right\}_{\mathbf{n}} \sim_{\sigma} f
$$

Moreover, if the first assumption is replaced by $\left\{B_{\mathbf{n}, m}\right\}_{\mathbf{n}} \sim_{\lambda} f_{m}$ for every $m$, given that the other two assumptions are left unchanged, and all the involved matrices are Hermitian, then $\left\{A_{\mathbf{n}}\right\}_{\mathbf{n}} \sim_{\lambda} f$.

In [5], a useful asymptotic spectral result is provided in the unilevel setting for matrix-sequences $\left\{Y_{n} T_{n}[f]\right\}_{n}$, where $Y_{n}$ is the anti-identity matrix.

TheOREM 2.7 ([5]). Let $Y_{n}$ be the $n \times n$ anti-identity matrix and $T_{n}[f]$ be the $n \times n$ Toeplitz matrix generated by a univariate Lebesgue integrable function $f \in L^{1}([-\pi, \pi])$. If $f$ has real Fourier coefficients, then the matrix-sequence $\left\{Y_{n} T_{n}[f]\right\}_{n}$ is distributed in eigenvalue sense as the function

$$
\psi_{|f|}(\theta)=\left\{\begin{array}{cc}
|f|(\theta), & \theta \in[0,2 \pi], \\
-|f|(\theta+2 \pi), & \theta \in[-2 \pi, 0)
\end{array} .\right.
$$

Our main goal is the generalization of the latter result for a $k$-variate $f \in L^{1}\left([-\pi, \pi]^{k}\right), k>1$. First, in Theorem 2.8, we provide a precise description of the asymptotic singular value distribution of $\left\{Y_{\mathbf{n}} T_{\mathbf{n}}[f]\right\}_{\mathbf{n}}$, where the real Toeplitz matrix $T_{\mathbf{n}}[f]$ is generated by $f \in L^{1}\left([-\pi, \pi]^{k}\right)$. Then, in Section 3 , we exploit it to provide an elegant description of the asymptotic spectral distribution of $\left\{Y_{\mathbf{n}} T_{\mathbf{n}}[f]\right\}_{\mathbf{n}}$.

THEOREM 2.8. Suppose $f \in L^{1}\left([-\pi, \pi]^{k}\right)$ with real Fourier coefficients and $Y_{\mathbf{n}} \in \mathbb{R}^{N(\mathbf{n}) \times N(\mathbf{n})}$ is the multilevel anti-identity matrix $Y_{\mathbf{n}}=Y_{n_{1}} \otimes \ldots \otimes Y_{n_{k}}=Y_{N(\mathbf{n})}$. Let $T_{\mathbf{n}}[f] \in \mathbb{R}^{N(\mathbf{n}) \times N(\mathbf{n})}$ be the Toeplitz matrix generated by $f$. Then

$$
\left\{Y_{\mathbf{n}} T_{\mathbf{n}}[f]\right\}_{\mathbf{n}} \sim_{\sigma} f
$$

Proof. Consider the (full) singular value decomposition of $T_{\mathbf{n}}[f]=U \Sigma V^{*}$, where $U, V$ are unitary matrices of size $N(\mathbf{n})$ and $\Sigma$ is the diagonal matrix containing the singular values $\sigma_{1}, \ldots, \sigma_{N(\mathbf{n})}$ of $T_{\mathbf{n}}[f]$.

We can write

$$
Y_{N(\mathbf{n})} T_{\mathbf{n}}[f]=\left(Y_{N(\mathbf{n})} U\right) \Sigma V^{*}
$$

Since $Y_{N(\mathbf{n})}$ is a unitary matrix, $Y_{N(\mathbf{n})} U$ is unitary and the previous formula is a singular value decomposition of $Y_{N(\mathbf{n})} T_{\mathbf{n}}[f]$. Hence, the sequence $\left\{Y_{N(\mathbf{n})} T_{\mathbf{n}}[f]\right\}_{\mathbf{n}}$ has the same singular value distribution of $\left\{T_{\mathbf{n}}[f]\right\}_{\mathbf{n}}$, which we know from Theorem 2.3. Consequently,

$$
\left\{Y_{\mathbf{n}} T_{\mathbf{n}}[f]\right\}_{\mathbf{n}} \sim_{\sigma} f
$$

and this completes the proof. 
3. Main results. In this section, we provide the main results on the spectral distribution of $\left\{Y_{\mathbf{n}} T_{\mathbf{n}}[f]\right\}_{\mathbf{n}}$.

First, we report a general tool useful for the latter purpose and we define the function that will have a crucial role in the description of the spectrum of $Y_{\mathbf{n}} T_{\mathbf{n}}[f]$.

Definition 3.1. Given the vector $\mathbf{p}=[2 \pi, 2 \pi, \ldots, 2 \pi]^{T} \in \mathbb{R}^{k}$ and a function $g$ defined over $[0,2 \pi]^{k}$, we define $\psi_{g}$ over $[-2 \pi, 0]^{k} \cup[0,2 \pi]^{k}$ in the following manner

$$
\psi_{g}(\boldsymbol{\theta})=\left\{\begin{array}{c}
g(\boldsymbol{\theta}), \\
-g(\boldsymbol{\theta}+\mathbf{p}), \quad \boldsymbol{\theta} \in[-2 \pi, 0]^{k}, \boldsymbol{\theta} \neq \mathbf{0}
\end{array} .\right.
$$

Theorem 3.2. Suppose $n \in \mathbb{Z}$ and $A(n) \in \mathbb{C}^{n \times n}$. Let $B_{n} \in \mathbb{C}^{2 n \times 2 n}$ be Hermitian matrices such that

$$
B_{n}=\left[\begin{array}{cc}
O & A(n) \\
A(n)^{H} & O
\end{array}\right]
$$

with $O$ being the square null matrices of size $n$. If $\sigma_{1}, \ldots, \sigma_{n}$ are the singular values of $A(n)$, then the eigenvalue of $B_{n}$ are given by $\pm \sigma_{j}, j=1, \ldots, n$.

In the following, we show that the spectral distribution of $\left\{Y_{\mathbf{n}} T_{\mathbf{n}}[f]\right\}_{\mathbf{n}}$ is described by $\psi_{|f|}(\boldsymbol{\theta})$ over the domain $[-2 \pi, 0]^{k} \cup[0,2 \pi]^{k}$. In particular, in Lemma 3.3 we prove that this holds for a trigonometric polynomial and in Theorem 3.4 we extend the result to a generic $f \in L^{1}\left([-\pi, \pi]^{k}\right)$.

LEMMA 3.3. Suppose that $f$ is a $k$-variate trigonometric polynomial of degree $\mathbf{r}=\left(r_{1}, r_{2}, \ldots, r_{k}\right)$ with real Fourier coefficients. Let $Y_{\mathbf{n}} \in \mathbb{R}^{N(\mathbf{n}) \times N(\mathbf{n})}$ be the anti-identity matrix $Y_{\mathbf{n}}=Y_{n_{1}} \otimes \ldots \otimes Y_{n_{k}}=Y_{N(\mathbf{n})}$ and let $T_{\mathbf{n}}[f] \in \mathbb{R}^{N(\mathbf{n}) \times N(\mathbf{n})}$ be the Toeplitz matrix generated by $f$. Then,

$$
\left\{Y_{\mathbf{n}} T_{\mathbf{n}}[f]\right\}_{\mathbf{n}} \sim_{\lambda} \psi_{|f|}
$$

over the domain $[-2 \pi, 0]^{k} \cup[0,2 \pi]^{k}$, where $\psi_{|f|}$ is given as in Definition 3.1 .

Proof. First, we assume that we are in the case $\mathbf{n}=\left(n_{1}, n_{2}, \ldots, n_{k}\right)$ with even $n_{1}=2 \nu_{1}$. The trigonometric polynomial $f$ can be written in terms of its Fourier coefficients as

$$
f(\boldsymbol{\theta})=\sum_{\mathbf{j}=-\mathbf{r}}^{\mathbf{r}} \hat{f}_{\mathbf{j}} \mathrm{e}^{\iota\langle\mathbf{j}, \boldsymbol{\theta}\rangle}
$$

where $\boldsymbol{\theta}=\left(\theta_{1}, \ldots, \theta_{k}\right),\langle\mathbf{j}, \boldsymbol{\theta}\rangle=\sum_{i=1}^{k} j_{i} \theta_{i}$.

Consider the following $2 \times 2$ block matrix-sequence

$$
\left\{M_{\mathbf{n}}\right\}_{\mathbf{n}}=\left\{\left[\begin{array}{cc}
O & Y_{\tilde{\mathbf{n}}} T_{\tilde{\mathbf{n}}}[f] \\
Y_{\tilde{\mathbf{n}}} T_{\tilde{\mathbf{n}}}[f] & O
\end{array}\right]\right\}_{\mathbf{n}}
$$

with blocks of dimension $N(\tilde{\mathbf{n}})=\nu_{1} n_{2} \ldots n_{k}, \tilde{\mathbf{n}}=\left(\nu_{1}, n_{2}, \ldots, n_{k}\right)$.

Due to its particular structure, we can easily obtain the asymptotic eigenvalue distribution of $\left\{M_{\mathbf{n}}\right\}_{n}$. Indeed Theorem 3.2 implies that the eigenvalues of $M_{\mathbf{n}}$ are $\pm \sigma_{j}\left(Y_{\tilde{\mathbf{n}}} T_{\tilde{\mathbf{n}}}[f]\right)= \pm \sigma_{j}\left(T_{\tilde{\mathbf{n}}}[f]\right), j=1, \ldots, N(\tilde{\mathbf{n}})$.

Consequently, we can conclude that

$$
\left\{M_{\mathbf{n}}\right\}_{\mathbf{n}} \sim_{\lambda} \psi_{|f|}
$$


Concerning the matrix $Y_{\mathbf{n}} T_{\mathbf{n}}[f]$, we note that it can be written as a $2 \times 2$ block matrix with block of size $N(\tilde{\mathbf{n}}) \times N(\tilde{\mathbf{n}})$. Indeed,

$$
Y_{\mathbf{n}} T_{\mathbf{n}}[f]=\left[\begin{array}{cc}
H_{\tilde{\mathbf{n}}}^{(1)} & Y_{\tilde{\mathbf{n}} T_{\tilde{\mathbf{n}}}[f]} \\
Y_{\tilde{\mathbf{n}}} T_{\tilde{\mathbf{n}}}[f] & H_{\tilde{\mathbf{n}}}^{(2)}
\end{array}\right]=M_{\mathbf{n}}+\left[\begin{array}{cc}
H_{\tilde{\mathbf{n}}}^{(1)} & O \\
O & H_{\tilde{\mathbf{n}}}^{(2)}
\end{array}\right],
$$

where $H_{\tilde{\mathbf{n}}}^{(1)}$ and $H_{\tilde{\mathbf{n}}}^{(2)}$ are particular multilevel Hankel matrices of size $N(\tilde{\mathbf{n}}) \times N(\tilde{\mathbf{n}})$.

To conclude, the proof we want to show that $\left\{Y_{\mathbf{n}} T_{\mathbf{n}}[f]\right\}_{\mathbf{n}},\left\{M_{\mathbf{n}}\right\}_{\mathbf{n}}$ and $\psi_{|f|}$ satisfy the hypotheses of Lemma 2.6, with $f_{m} \equiv \psi_{|f|},\left\{A_{\mathbf{n}}\right\}_{\mathbf{n}} \equiv\left\{M_{\mathbf{n}}\right\}_{\mathbf{n}}$ and $\left\{B_{\mathbf{n}, m}\right\}_{\mathbf{n}} \equiv\left\{Y_{\mathbf{n}} T_{\mathbf{n}}[f]\right\}_{\mathbf{n}}$. That is, $\left\{Y_{\mathbf{n}} T_{\mathbf{n}}[f]\right\}_{\mathbf{n}}$ is a constant class of sequences that is not depending on the variable $m$. In particular, it is sufficient to verify that

$$
\left\{Y_{\mathbf{n}} T_{\mathbf{n}}[f]\right\}_{\mathbf{n}} \stackrel{\text { a.c.s. wrt } m}{\longrightarrow}\left\{M_{\mathbf{n}}\right\}_{\mathbf{n}},
$$

since we already have relation (3.3) and obviously $\left\{\psi_{|f|}\right\}_{m} \rightarrow \psi_{|f|}$ in measure.

Consequently, according to the Definition 2.4, it is sufficient to prove that the matrix-sequence

$$
\left\{E_{\mathbf{n}}\right\}_{\mathbf{n}}=\left\{\left[\begin{array}{cc}
H_{\tilde{\mathbf{n}}}^{(1)} & O \\
O & H_{\tilde{\mathbf{n}}}^{(2)}
\end{array}\right]\right\}_{\mathbf{n}},
$$

is such that for $\mathbf{n} \geq \mathbf{n}_{m}$

$$
\operatorname{rank} E_{\mathbf{n}} \leq c(m) N(\mathbf{n}),
$$

where $c(m)$ depends only on $m$ and $\lim _{m \rightarrow \infty} c(m)=0$.

The fact that the $f$ is a trigonometric polynomial of degree $\mathbf{r}$ implies that

$$
\operatorname{rank} H_{\tilde{\mathbf{n}}}^{(i)} \leq r_{1} n_{2} n_{3} \ldots n_{k}, \quad i=1,2
$$

Then, for $\mathbf{n} \geq \mathbf{n}_{m}$,

$$
\operatorname{rank}\left(E_{\mathbf{n}}\right) \leq 2 r_{1} n_{2} n_{3} \ldots n_{k}=\frac{2 r_{1} m}{m} n_{2} n_{3} \ldots n_{k} .
$$

and so, for $\mathbf{n}$ such that $n_{1} \geq 2 r_{1} m$, we obtain

$$
\operatorname{rank}\left(E_{\mathbf{n}}\right) \leq \frac{1}{m} N(\mathbf{n}), \quad \text { with } \lim _{m \rightarrow \infty} \frac{1}{m}=0 .
$$

For the case where $n_{1}$ is odd, the proof is of the same type as before with a few slight technical changes in the decomposition in (3.4). Indeed, the result of Theorem 3.2 is maintained also for odd dimensions. Then, is possible to follow an analogous proof as it has been done for the unilevel case in [5, Theorem 3.2]

THEOREM 3.4. Suppose $f \in L^{1}\left([-\pi, \pi]^{k}\right)$ a $k$-variate function with real Fourier coefficients, periodically extended to the whole real plane. Let $Y_{\mathbf{n}} \in \mathbb{R}^{N(\mathbf{n}) \times N(\mathbf{n})}$ be the anti-identity matrix $Y_{\mathbf{n}}=Y_{n_{1}} \otimes \ldots \otimes Y_{n_{k}}=Y_{N(\mathbf{n})}$ and let $T_{\mathbf{n}}[f] \in \mathbb{R}^{N(\mathbf{n}) \times N(\mathbf{n})}$ be the Toeplitz matrix generated by $f$. Then,

$$
\left\{Y_{\mathbf{n}} T_{\mathbf{n}}[f]\right\}_{\mathbf{n}} \sim_{\lambda} \psi_{|f|}
$$

over the domain $[-2 \pi, 0]^{k} \cup[0,2 \pi]^{k}$, where $\psi_{|f|}$ is given as in Definition 3.1. 
Proof. Since the set of $k$ th variate trigonometric polynomials is dense in $L^{1}\left([-\pi, \pi]^{k}\right)$, there exists a sequence $\left\{f_{m}\right\}_{m}$ of trigonometric polynomials such that $\left\{f_{m}\right\}_{m} \rightarrow f$ in $L^{1}\left([-\pi, \pi]^{k}\right)$.

From Lemma 2.6, we obtain that $\left\{Y_{\mathbf{n}} T_{\mathbf{n}}[f]\right\}_{\mathbf{n}} \sim_{\lambda} \psi_{|f|}$ if the sequences $\left\{Y_{\mathbf{n}} T_{\mathbf{n}}[f]\right\}_{\mathbf{n}},\left\{Y_{\mathbf{n}} T_{\mathbf{n}}\left[f_{m}\right]\right\}_{\mathbf{n}}$ and the functions $\psi_{|f|}$ and $\psi_{\left|f_{m}\right|}$ satisfy the following:

- $\left\{Y_{\mathbf{n}} T_{\mathbf{n}}\left[f_{m}\right]\right\}_{\mathbf{n}} \sim_{\lambda} \psi_{\left|f_{m}\right|}$

- $\left\{Y_{\mathbf{n}} T_{\mathbf{n}}\left[f_{m}\right]\right\}_{\mathbf{n}} \stackrel{\text { a.c.s. wrt } m}{\longrightarrow}\left\{Y_{\mathbf{n}} T_{\mathbf{n}}(f)\right\}_{\mathbf{n}}$;

- $\left\{\psi_{\left|f_{m}\right|}\right\}_{m} \rightarrow \psi_{|f|}$ in measure.

The first item is a consequence of Lemma 3.3 , since each $f_{m}$ is a multivariate trigonometric polynomial.

For the second item, we use the characterization Theorem 2.5. In particular, using the linearity of the operator $T_{\mathbf{n}}(\cdot)$, we find $T_{\mathbf{n}}(f)-T_{\mathbf{n}}\left(f_{m}\right)=T_{\mathbf{n}}\left(f-f_{m}\right)$ and hence

$$
\left\|Y_{\mathbf{n}} T_{\mathbf{n}}(f)-Y_{\mathbf{n}} T_{\mathbf{n}}\left(f_{m}\right)\right\|_{1}=\left\|T_{\mathbf{n}}\left(f-f_{m}\right)\right\|_{1},
$$

because $Y_{\mathbf{n}}$ is unitary and $\|\cdot\|_{1}$ is a unitarily invariant norm (see [2] for a remarkably rich treatment of unitarily invariant norms). Furthermore, as proven in [22] using the characterizations of the unitarily invariant norms [2], we have

$$
\left\|Y_{\mathbf{n}}\right\|\left\|T_{\mathbf{n}}\left(f-f_{m}\right)\right\|_{1} \leq \frac{N(\mathbf{n})}{(2 \pi)^{k}}\left\|f-f_{m}\right\|_{L^{1}}
$$

so that

$$
\left\|Y_{\mathbf{n}} T_{\mathbf{n}}(f)-Y_{\mathbf{n}} T_{\mathbf{n}}\left(f_{m}\right)\right\|_{1} \leq \frac{N(\mathbf{n})}{(2 \pi)^{k}}\left\|f-f_{m}\right\|_{L^{1}} .
$$

Since $\lim _{m \rightarrow \infty}\left\|f-f_{m}\right\|_{L^{1}}=0$, we conclude that

$$
\left\{Y_{\mathbf{n}} T_{\mathbf{n}}\left[f_{m}\right]\right\}_{\mathbf{n}} \stackrel{\text { a.c.s. wrt } m}{\longrightarrow}\left\{Y_{\mathbf{n}} T_{\mathbf{n}}(f)\right\}_{\mathbf{n}} .
$$

Finally, we prove that $\left\{\psi_{\left|f_{m}\right|}\right\}_{m} \rightarrow \psi_{|f|}$ in $L^{1}\left([-\pi, \pi]^{k}\right)$, so that the convergence in measure is a direct consequence.

In particular, we have to prove that

$$
\lim _{m \rightarrow \infty} \int_{\Omega}\left|\psi_{|f|}-\psi_{\left|f_{m}\right|}\right| \mathrm{d} \boldsymbol{\theta}=0,
$$

where $\Omega=[-2 \pi, 0]^{k} \cup[0,2 \pi]^{k}$. Since $f$ and $f_{m}$ are $2 \pi$-periodic functions, it holds

$$
\begin{aligned}
\int_{\Omega}\left|\psi_{|f|}-\psi_{\left|f_{m}\right|}\right| \mathrm{d} \boldsymbol{\theta}= & \int_{(0,2 \pi]^{k}}|| f|-| f_{m}|| \mathrm{d} \boldsymbol{\theta}+\int_{[-2 \pi, 0)^{k}}|-| f|+| f_{m}|| \mathrm{d} \boldsymbol{\theta}= \\
& 2 \int_{[-\pi, \pi]^{k}}|| f|-| f_{m}|| \mathrm{d} \boldsymbol{\theta} \leq 2 \int_{[-\pi, \pi]^{k}}\left|f-f_{m}\right| \mathrm{d} \boldsymbol{\theta} .
\end{aligned}
$$

Then, since $\left\{f_{m}\right\}_{m} \rightarrow f$ in $L^{1}\left([-\pi, \pi]^{k}\right)$,

$$
\lim _{m \rightarrow \infty} \int_{\Omega}\left|\psi_{|f|}-\psi_{\left|f_{m}\right|}\right| \mathrm{d} \boldsymbol{\theta} \leq \lim _{m \rightarrow \infty} 2 \int_{[-\pi, \pi]^{k}}\left|f-f_{m}\right| \mathrm{d} \boldsymbol{\theta}=0
$$

which implies that $\left\{\psi_{\left|f_{m}\right|}\right\}_{m} \rightarrow \psi_{|f|}$ in measure. 
Note that $\psi_{|f|}$ is not the unique function which describes the asymptotic spectral distribution of $\left\{Y_{\mathbf{n}} T_{\mathbf{n}}[f]\right\}_{\mathbf{n}}$. Indeed, it is possible to find a rearrangement $\phi_{|f|}$ of $\psi_{|f|}$ (and viceversa) such that $\left\{Y_{\mathbf{n}} T_{\mathbf{n}}[f]\right\}_{\mathbf{n}} \sim_{\lambda} \phi_{|f|}$, see Corollary 3.5. In addition, under the hypotheses of separability of $f$, we can construct a spectral symbol $h_{f}$ with a tensor product argument, see Proposition 4.1.

COROLlary 3.5. Suppose $f \in L^{1}\left([-\pi, \pi]^{k}\right)$ is a $k$-variate function with real Fourier coefficients, periodically extended to the whole real plane. Let $Y_{\mathbf{n}}=Y_{n_{1}} \otimes \ldots \otimes Y_{n_{k}}=Y_{N(\mathbf{n})} \in \mathbb{R}^{N(\mathbf{n}) \times N(\mathbf{n})}$ be the anti-identity matrix. Let $T_{\mathbf{n}}[f] \in \mathbb{R}^{N(\mathbf{n}) \times N(\mathbf{n})}$ be the Toeplitz matrix generated by $f$. Then,

$$
\left\{Y_{\mathbf{n}} T_{\mathbf{n}}[f]\right\}_{\mathbf{n}} \sim_{\lambda} \phi_{|f|}
$$

over the domain $[-2 \pi, 0]^{k} \cup[0,2 \pi]^{k}$ with $\phi_{g}$ defined in the following way

$$
\phi_{g}(\boldsymbol{\theta})=\left\{\begin{array}{c}
g(\boldsymbol{\theta}), \quad \boldsymbol{\theta} \in[0,2 \pi]^{k} \\
-g(-\boldsymbol{\theta}), \quad \boldsymbol{\theta} \in[-2 \pi, 0)^{k}
\end{array}\right.
$$

Proof. We observe that for any $F$ continuous with bounded support

$$
\int_{[-2 \pi, 2 \pi]^{k}} F\left(\phi_{|f|}\right) \mathrm{d} \boldsymbol{\theta}=\int_{[-2 \pi, 2 \pi]^{k}} F\left(\psi_{|f|}\right) \mathrm{d} \boldsymbol{\theta},
$$

i.e. $\phi_{|f|}$ is a rearrangement of $\psi_{|f|}$ (and viceversa) [7, Section 3.2]. Hence, by the very definition of distribution, we have $\left\{Y_{\mathbf{n}} T_{\mathbf{n}}[f]\right\}_{\mathbf{n}} \sim_{\lambda} \phi_{|f|}$ if and only if $\left\{Y_{\mathbf{n}} T_{\mathbf{n}}[f]\right\}_{\mathbf{n}} \sim_{\lambda} \psi_{|f|}$. Therefore, the desired result is an immediate consequence of Theorem 3.4.

4. Further results and remarks. The distribution results can be combined and complemented with analogous studies on the preconditioned matrix-sequences: in this sense, the paper by Pestana already is a step in this direction, that is, in the multilevel setting (refer to [16]).

Furthermore, the proof techniques employed so far can be easily extended to the case of a generating function which is multivariate and matrix-valued, so covering the multilevel block setting as done in [5] for the univariate case.

The case of separable generating functions deserves particular attention because one has beautiful tensor structures and hence the distributional results in the multilevel context can be directly deduced by the unilevel case, as shown in some detail in the next result.

Proposition 4.1. Suppose $f \in L^{1}\left([-\pi, \pi]^{k}\right)$ is a $k$-variate function periodically extended to the whole real plane. Assume that $f$ is $k$-separable, that is, there exist $k$ functions $f_{i} \in L^{1}([-\pi, \pi]), i=1, \ldots, k$, such that $f=f_{1} \otimes \cdots \otimes f_{k}$. Suppose that the functions $f_{i}, i=1, \ldots, k$, have real Fourier coefficients. Let $Y_{\mathbf{n}}=Y_{n_{1}} \otimes \ldots \otimes Y_{n_{k}}=Y_{N(\mathbf{n})} \in \mathbb{R}^{N(\mathbf{n}) \times N(\mathbf{n})}$ be the anti-identity matrix. Let $T_{\mathbf{n}}[f] \in \mathbb{R}^{N(\mathbf{n}) \times N(\mathbf{n})}$ be the Toeplitz matrix generated by $f$. Then,

$$
\left\{Y_{\mathbf{n}} T_{\mathbf{n}}[f]\right\}_{\mathbf{n}} \sim_{\lambda} h_{f}
$$

over the domain $[-2 \pi, 2 \pi]^{k}$, where the function $h_{f}$ is defined as

$$
h_{f}=\psi_{\left|f_{1}\right|} \otimes \cdots \otimes \psi_{\left|f_{k}\right|},
$$

and $\psi_{\left|f_{i}\right|}, i=1, \ldots, k$, is defined as in equation (2.1). Furthermore, $\psi_{|f|}, \phi_{|f|}$ are both rearrangements of $h_{f}$ (and viceversa). 
Electronic Journal of Linear Algebra, ISSN 1081-3810

A publication of the International Linear Algebra Society

Volume 37, pp. 370-386, April 2021.

Proof. From Lemma 2.1 and the definition of $Y_{\mathbf{n}}$, we obtain

$$
Y_{\mathbf{n}} T_{\mathbf{n}}[f]=Y_{\mathbf{n}} T_{\mathbf{n}}\left[f_{1} \otimes \cdots \otimes f_{k}\right]=\left(Y_{n_{1}} \otimes \cdots \otimes Y_{n_{k}}\right)\left(T_{n_{1}}\left[f_{1}\right] \otimes \cdots \otimes T_{n_{k}}\left[f_{k}\right]\right)
$$

From the mixed product property of the Kronecker product, we have

$$
Y_{\mathbf{n}} T_{\mathbf{n}}[f]=Y_{n_{1}} T_{n_{1}}\left[f_{1}\right] \otimes \cdots \otimes Y_{n_{k}} T_{n_{k}}\left[f_{k}\right]
$$

From Theorem 2.7, the following spectral asymptotic results hold for $j=1, \ldots, k$

$$
\left\{Y_{n_{j}} T_{n_{j}}\left[f_{j}\right]\right\}_{n_{j}} \sim_{\lambda} \psi_{\left|f_{i}\right|}
$$

Hence, the spectrum of $\left\{Y_{\mathbf{n}} T_{\mathbf{n}}[f]\right\}_{\mathbf{n}}$ is asymptotically described by the tensor products of each $\psi_{\left|f_{i}\right|}, i=$ $1, \ldots, k$. That is,

$$
\left\{Y_{\mathbf{n}} T_{\mathbf{n}}[f]\right\}_{\mathbf{n}} \sim_{\lambda} \psi_{\left|f_{1}\right|} \otimes \cdots \otimes \psi_{\left|f_{k}\right|}=h_{f}
$$

Finally, by combining the last equation with Theorem 3.4 and Corollary 3.5, by virtue of the definition of spectral distribution, we deduce that the functions $\psi_{|f|}, \phi_{|f|}$ are necessarily both rearrangements of $h_{f}$ (and viceversa).

Note that a $k$-variate trigonometric polynomial of degree $\mathbf{r}=\left(r_{1}, r_{2}, \ldots, r_{k}\right)$ with real Fourier coefficients $\hat{f}_{\mathbf{j}}$ has the explicit form

$$
f(\boldsymbol{\theta})=\sum_{j_{1}=-r_{1}}^{r_{1}} \sum_{j_{2}=-r_{2}}^{r_{2}} \cdots \sum_{j_{k}=-r_{k}}^{r_{k}} \hat{f}_{j_{1}, j_{2}, \ldots, j_{k}} \mathrm{e}^{\iota \sum_{i=1}^{k} j_{i} \theta_{i}}
$$

That is, it can be written as finite sums of separable trigonometric polynomial $\left\{\hat{f}_{j_{1}, j_{2}, 1 \ldots, j_{k}} \mathrm{e}^{\iota \sum_{i=1}^{k} j_{i} \theta_{i}}\right\}_{j_{1}, j_{2}, \ldots, j_{k}}$. Hence, on each term, we can apply Lemma 2.1 and obtain, for $-\mathbf{r} \leq \mathbf{j} \leq \mathbf{r}$,

$$
\left\{Y_{\mathbf{n}} T_{\mathbf{n}}\left[\hat{f}_{j_{1}, j_{2}, \ldots, j_{k}} \mathrm{e}^{\iota \sum_{i=1}^{k} j_{i} \theta_{i}}\right]\right\}_{\mathbf{n}} \sim_{\lambda} \hat{f}_{j_{1}, j_{2}, \ldots, j_{k}}\left(\psi_{\mid \mathrm{e}^{\iota j_{1} \theta_{1} \mid}} \otimes \cdots \otimes \psi_{\left|\mathrm{e}^{\iota j_{k}} \theta_{k}\right|}\right)
$$

Therefore, an interesting further investigation is the study of the stability of the spectral distribution for the sum in (4.7): more specifically, is it true that $\left\{Y_{\mathbf{n}} T_{\mathbf{n}}\left[g_{j}\right]\right\}_{\mathbf{n}} \sim_{\lambda} g_{j}, g_{j}$ separable, $j=1, \ldots, t$, implies $\left\{Y_{\mathbf{n}} T_{\mathbf{n}}[f]\right\}_{\mathbf{n}} \sim_{\lambda} f$ with $f$ being any sum of the separable terms $g_{j}, j=1, \ldots, t$ ? In fact, such a proof would lead to a simplification when treating distribution results for generic multilevel matrix-sequences. Finally, it is worth mentioning that all the results in Theorem 3.4, Corollary 3.5, Proposition 4.1 also hold in the version for singular values for a very basic reason, so that

$$
\left\{Y_{\mathbf{n}} T_{\mathbf{n}}[f]\right\}_{\mathbf{n}} \sim_{\sigma} \psi_{|f|}, \quad\left\{Y_{\mathbf{n}} T_{\mathbf{n}}[f]\right\}_{\mathbf{n}} \sim_{\sigma} \phi_{|f|}, \quad\left\{Y_{\mathbf{n}} T_{\mathbf{n}}[f]\right\}_{\mathbf{n}} \sim_{\sigma} h_{f}
$$

respectively. In reality, since $Y_{\mathbf{n}} T_{\mathbf{n}}[f]$ is real symmetric for every $\mathbf{n}$, by comparing the singular value decomposition and the spectral decomposition, it is immediate to see that the singular values of $Y_{\mathbf{n}} T_{\mathbf{n}}[f]$ are the modulus of corresponding eigenvalues. As consequence, taking into account Theorem 2.8, we infer that $\left|\psi_{|f|}\right|,\left|\phi_{|f|}\right|,\left|h_{f}\right|$ are all rearrangements of $|f|$. 
5. Numerical experiments. In this section, we numerically show that the statements of Theorem 3.4 and Proposition 4.1 are true in the cases of both bivariate trigonometric polynomials and generic separable functions in $L^{1}\left([-\pi, \pi]^{2}\right)$. In particular, we illustrate the predicted behavior of the eigenvalues for the matrix-sequences $\left\{Y_{\mathbf{n}} T_{\mathbf{n}}[f]\right\}_{\mathbf{n}}$ for a function $f$ in the following cases.

- Example 1. $f$ is a bivariate trigonometric polynomial $f:[-\pi, \pi]^{2} \mapsto \mathbb{C}$ with high degree.

- Example 2. $f$ is the bivariate separable trigonometric polynomial $f:[-\pi, \pi]^{2} \mapsto \mathbb{C}$,

$$
f\left(\theta_{1}, \theta_{2}\right)=\left(10-3 \mathrm{e}^{\iota\left(\theta_{1}\right)}+\mathrm{e}^{\iota\left(-\theta_{1}\right)}\right)\left(4-3 \mathrm{e}^{\iota\left(\theta_{2}\right)}\right) .
$$

- Example 3. $f \in L^{1}\left([-\pi, \pi]^{2}\right)$ is the separable function $f\left(\theta_{1}, \theta_{2}\right)=\theta_{1}^{2} \theta_{2}^{2}$.

In order to numerically support the validity of the asymptotic spectral distribution provided by Theorem 3.4 (resp. Proposition 4.1), we show that for large enough $n$ the eigenvalues of $Y_{\mathbf{n}} T_{\mathbf{n}}[f]$ are approximately equal to the samples of $\psi_{|f|}$ (resp. $h_{f}$ ) over a uniform grid. We remark that the theory admits the possible exception of outliers, whose number is infinitesimal respect to the dimension $N(\mathbf{n})$ of the matrix.

Moreover, since the matrices $Y_{\mathbf{n}} T_{\mathbf{n}}[f]$ are symmetric for any $\mathbf{n}$, the values $\lambda_{j}\left(Y_{\mathbf{n}} T_{\mathbf{n}}[f]\right)$ are real for $j=1, \ldots, N(\mathbf{n})$ and, hence, they can be ordered. Precisely, if $\mathbf{n}=\left(n_{1}, n_{2}\right)$, we adopt the following procedure. First, we evaluate the function $\psi_{|f|}\left(\right.$ resp. $\left.h_{f}\right)$ on a chosen uniform grid $\tau_{j_{1}, j_{2}}^{n_{1}, n_{2}}, j_{1}=1, \ldots, n_{1}, j_{2}=1, \ldots, n_{2}$, and we save the evaluations in a $n_{1} \times n_{2}$ matrix $M=\left[M_{j_{1}, j_{2}}\right]$. Then, we order them in ascending order using the $\operatorname{sort}(\cdot)$ function of MATLAB version 9.6.0.1099231 (R2019a) applied to $M$, which also returns a matrix $\mathcal{I}$ of indices which specifies how the elements of $M$ were rearranged to obtain the sorted output column by column. Finally, we order the values $\lambda_{j}\left(Y_{\mathbf{n}} T_{\mathbf{n}}[f]\right), j=1, \ldots, n_{1} n_{2}$, according to the vectorization of $\mathcal{I}$ and we reshape them in matrix form. This procedure ensures a unique and natural correspondence between the eigenvalues and the samplings over $\tau_{j_{1}, j_{2}}^{n_{1}, n_{2}}$, even in presence of outliers. When we exploit such ordering in the following examples, we say that the eigenvalues are sorted according to the order given by $\psi_{|f|}$ (resp. $h_{f}$ ) over $\tau_{j_{1}, j_{2}}^{n_{1}, n_{2}}$.

EXAMPLE 5.1. We consider the bivariate trigonometric polynomial $f:[-\pi, \pi]^{2} \mapsto \mathbb{C}$ defined by

$$
\begin{aligned}
f\left(\theta_{1}, \theta_{2}\right)= & 5+\mathrm{e}^{\iota\left(\theta_{1}\right)}+\mathrm{e}^{\iota\left(2 \theta_{1}\right)}-3 \mathrm{e}^{\iota\left(-\theta_{1}\right)}+2 \mathrm{e}^{\iota\left(-2 \theta_{1}\right)}+2 \mathrm{e}^{\iota\left(\theta_{2}\right)}+\mathrm{e}^{\iota\left(2 \theta_{2}\right)}-2 \mathrm{e}^{\iota\left(-\theta_{2}\right)}+\mathrm{e}^{\iota\left(-2 \theta_{2}\right)} \\
& +\mathrm{e}^{\iota\left(\theta_{1}+\theta_{2}\right)}+3 \mathrm{e}^{\iota\left(\theta_{1}-\theta_{2}\right)}+3 \mathrm{e}^{\iota\left(-\theta_{1}+\theta_{2}\right)}+2 \mathrm{e}^{\iota\left(-\theta_{1}-\theta_{2}\right)}+\mathrm{e}^{\iota\left(-2 \theta_{1}+2 \theta_{2}\right)}+\mathrm{e}^{\iota\left(\theta_{1}+2 \theta_{2}\right)}+2 \mathrm{e}^{\iota\left(-2 \theta_{1}+\theta_{2}\right)} \\
& +3 \mathrm{e}^{\iota\left(2 \theta_{1}+\theta_{2}\right)}+\mathrm{e}^{\iota\left(-2 \theta_{1}-\theta_{2}\right)}+\mathrm{e}^{\iota\left(2 \theta_{1}-\theta_{2}\right)}+\mathrm{e}^{\iota\left(-2 \theta_{1}-2 \theta_{2}\right)}+\mathrm{e}^{\iota\left(-\theta_{1}-2 \theta_{2}\right)}+\mathrm{e}^{\iota\left(\theta_{1}-2 \theta_{2}\right)}+\mathrm{e}^{\iota\left(2 \theta_{1}-2 \theta_{2}\right)} .
\end{aligned}
$$

Hence, $f$ is a trigonometric polynomial with real Fourier coefficients. In particular, the coefficients of $f$ can be represented in a more compact and elegant form as a $2 D$ stencil

$$
\left[\begin{array}{ccccc}
1 & 1 & 1 & 3 & 0 \\
1 & 3 & 1 & 1 & 1 \\
1 & -2 & 5 & 2 & 1 \\
1 & 2 & -3 & 3 & 0 \\
1 & 1 & 2 & 2 & 1
\end{array}\right]
$$

Hence, Theorem 3.4 implies that the eigenvalues of $Y_{\mathbf{n}} T_{\mathbf{n}}[f]$ (properly sorted) are approximately equal to the samples of $\psi_{|f|}$ over the following grid $\xi_{j_{1}, j_{2}}^{\left(n_{1}, n_{2}\right)}$ of the domain $[-2 \pi, 0]^{2} \cup[0,2 \pi]^{2}$ 
Electronic Journal of Linear Algebra, ISSN 1081-3810

A publication of the International Linear Algebra Society

Volume 37, pp. 370-386, April 2021.

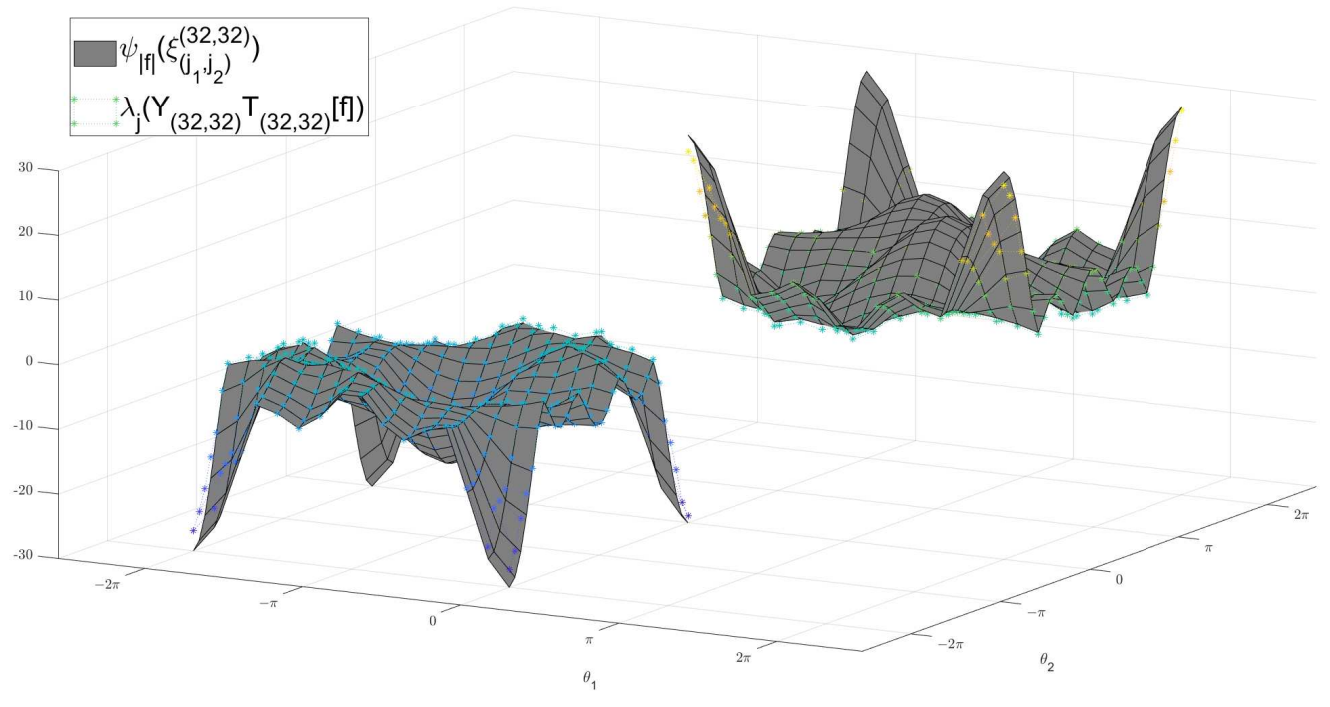

FIGURE 1. Example 1, a comparison between the eigenvalues $\lambda_{j}\left(Y_{(32,32)} T_{(32,32)}[f]\right)$ and the samples $\psi_{|f|}$ over the grid $\xi_{\left(j_{1}, j_{2}\right)}^{(32,32)}$, where $f$ is defined by (5.9).

$$
\xi_{j_{1}, j_{2}}^{\left(n_{1}, n_{1}\right)}=\gamma_{j_{1}, j_{2}}^{\left(n_{1}, n_{2}\right)} \cup\left(\gamma_{j_{1}, j_{2}}^{\left(n_{1}, n_{2}\right)}-2 \pi\right)
$$

where

$$
\gamma_{j_{1}, j_{2}}^{\left(n_{1}, n_{2}\right)}=2 \pi \frac{\left(j_{1}, j_{2}\right)}{\left(\frac{n_{1}}{2}-1, n_{2}-1\right)}, \quad j_{1}=0, \ldots, \frac{n_{1}}{2}-1, \quad j_{2}=0, \ldots, n_{2}-1
$$

We fix $n_{1}=n_{2}=32$. Then, $Y_{\mathbf{n}} T_{\mathbf{n}}[f] \in \mathbb{R}^{N(\mathbf{n}) \times N(\mathbf{n})}$ is a $N(\mathbf{n}) \times N(\mathbf{n})$ real symmetric matrix with eigenvalues $\lambda_{j}\left(Y_{\mathbf{n}} T_{\mathbf{n}}[f]\right), j=1, \ldots, N(\mathbf{n})$ and $N(\mathbf{n})=1024$. In Figure 1, we plot (in gray) the samples of $\psi_{|f|}$ over the grid $\xi_{j_{1}, j_{2}}^{(32,32)}$, and we can observe that they approximate without outliers the eigenvalues (colored dots) $\lambda_{j}\left(Y_{(32,32)} T_{(32,32)}[f]\right), j=1, \ldots, 1024$ sorted according to the order given by $\psi_{|f|}$ over $\xi_{j_{1}, j_{2}}^{(32,32)}$.

ExAmPLE 5.2. We consider the bivariate separable trigonometric polynomial $f:[-\pi, \pi]^{2} \mapsto \mathbb{C}$

$$
f\left(\theta_{1}, \theta_{2}\right)=\left(10-3 \mathrm{e}^{\iota\left(\theta_{1}\right)}+\mathrm{e}^{\iota\left(-\theta_{1}\right)}\right)\left(4-3 \mathrm{e}^{\iota\left(\theta_{2}\right)}\right)
$$

In particular, $f\left(\theta_{1}, \theta_{2}\right)$ can be decomposed, analogously as $f$ of Proposition 4.1, as the tensor product of univariate polynomials $f_{1}\left(\theta_{1}\right)=10-3 \mathrm{e}^{\iota\left(\theta_{1}\right)}+\mathrm{e}^{\iota\left(-\theta_{1}\right)}$ and $f_{2}\left(\theta_{2}\right)=4-3 \mathrm{e}^{\iota\left(\theta_{2}\right)}$. Moreover, it has the stencil given by

$$
\left[\begin{array}{ccc}
0 & -12 & -3 \\
0 & 40 & -30 \\
0 & 4 & 9
\end{array}\right]=\left[\begin{array}{c}
-3 \\
10 \\
1
\end{array}\right]\left[\begin{array}{lll}
0 & 4 & -3
\end{array}\right]
$$


This implies that we can provide two equivalent spectral distributions for the sequence $\left\{Y_{\mathbf{n}} T_{\mathbf{n}}[f]\right\}_{\mathbf{n}}$. Indeed, on the one hand, $f$ is a trigonometric polynomial with real Fourier coefficients, then, from Theorem 3.4

$$
\left\{Y_{\mathbf{n}} T_{\mathbf{n}}[f]\right\}_{\mathbf{n}} \sim_{\lambda} \psi_{|f|}
$$

where $\psi_{|f|}$ is defined as in formula (3.2). On the other hand, $f$ is a separable trigonometric polynomial satisfying the hypotheses of Proposition 4.1. Then,

$$
\left\{Y_{\mathbf{n}} T_{\mathbf{n}}[f]\right\}_{\mathbf{n}} \sim_{\lambda} h_{f}
$$

where $h_{f}$ is defined as in (4.6).

In Figure 2, we numerically show that both the results are accurate in approximating the eigenvalues of $Y_{\mathbf{n}} T_{\mathbf{n}}[f]$, since $\psi_{|f|}$ is a rearrangement of $h_{f}$ and viceversa. In particular, on the top of Figure 2, we can observe that the eigenvalues $\lambda_{j}\left(Y_{\mathbf{n}} T_{\mathbf{n}}[f]\right), j=1, \ldots, N(\mathbf{n}), \mathbf{n}=(32,32)$ are well approximated by the samplings of the function $\psi_{|f|}$ over the grid $\xi_{\left(j_{1}, j_{2}\right)}^{\left(n_{1}, n_{2}\right)}$. The grid $\xi_{\left(j_{1}, j_{2}\right)}^{\left(n_{1}, n_{2}\right)}$ of the domain $[-2 \pi, 0]^{2} \cup[0,2 \pi]^{2}$ is defined as in (5.11). Analogously, the bottom of Figure 2 numerically confirms that an accurate approximation of the eigenvalues $\lambda_{j}\left(Y_{\mathbf{n}} T_{\mathbf{n}}[f]\right), j=1, \ldots, N(\mathbf{n}), \mathbf{n}=(32,32)$ can be computed by a uniform sampling of the function $h_{f}=\psi_{\left|f_{1}\right|} \otimes \psi_{\left|f_{2}\right|}$ over the domain $[-2 \pi, 2 \pi]^{2}$. The uniform grid over $[-2 \pi, 2 \pi]^{2}$ is $\theta_{\left(j_{1}, j_{2}\right)}^{(32,32)}$ where

$$
\theta_{\left(j_{1}, j_{2}\right)}^{\left(n_{1}, n_{2}\right)}=4 \pi \frac{\left(j_{1}, j_{2}\right)}{\left(n_{1}-1, n_{2}-1\right)}-2 \pi, \quad j_{1}=0, \ldots, n_{1}-1, \quad j_{2}=0, \ldots, n_{2}-1
$$

EXAMPLE 5.3. In the last example, we consider the function $f:[-\pi, \pi]^{2} \rightarrow \mathbb{R}$ by

$$
f\left(\theta_{1}, \theta_{2}\right)=\theta_{1}^{2} \theta_{2}^{2}
$$

periodically extended to the real plane.

The function $f$ is not a trigonometric polynomial, and consequently, the matrices $T_{\mathbf{n}}[f]$ are dense, for all $\mathbf{n}$. Note that $f$ is a bivariate separable function in $L^{1}\left([-2 \pi, 2 \pi]^{2}\right)$ obtained as the tensor product of univariate functions with real Fourier coefficients.

In particular, the Fourier coefficients of the univariate function $\theta^{2}$ are given by the formulae

$$
\left\{\begin{array}{l}
a_{0}=\frac{\pi^{2}}{3}, \\
a_{k}=(-1)^{k} \frac{2}{k^{2}}, \quad k= \pm 1, \pm 2, \ldots .
\end{array} .\right.
$$

Recalling that $f$ is defined on $[-\pi, \pi]^{2}$ and periodically extended to the real plane, we can write the following explicit formulae for $f$ in $[0,2 \pi]^{2}$ :

$$
\begin{cases}\theta_{1}^{2} \theta_{2}^{2}, & \left(\theta_{1}, \theta_{2}\right) \in[0, \pi]^{2}, \\ \theta_{1}^{2}\left(\theta_{2}-2 \pi\right)^{2}, & \left(\theta_{1}, \theta_{2}\right) \in[0, \pi] \times(\pi, 2 \pi], \\ \left(\theta_{1}-2 \pi\right)^{2} \theta_{2}^{2}, & \left(\theta_{1}, \theta_{2}\right) \in(\pi, 2 \pi] \times[0, \pi], \\ \left(\theta_{1}-2 \pi\right)^{2}\left(\theta_{2}-2 \pi\right)^{2}, & \left(\theta_{1}, \theta_{2}\right) \in(\pi, 2 \pi]^{2} .\end{cases}
$$


Electronic Journal of Linear Algebra, ISSN 1081-3810

A publication of the International Linear Algebra Society

Volume 37, pp. 370-386, April 2021.
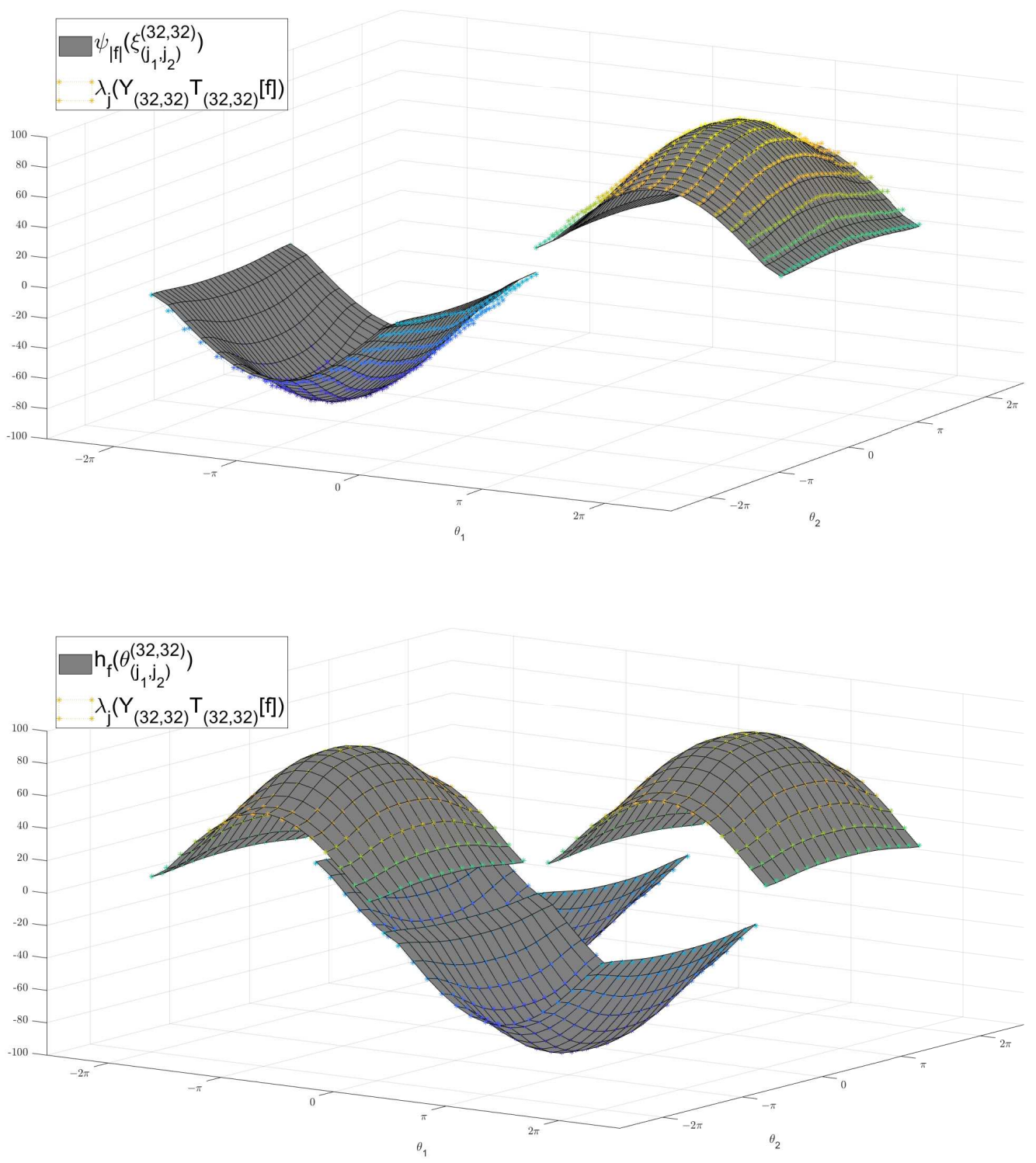

Figure 2. Example 5.2, a comparison between the eigenvalues $\lambda_{j}\left(Y_{(32,32)} T_{(32,32)}[f]\right)$ and (top) the samples $\psi_{|f|}$ over the $\operatorname{grid} \xi_{\left(j_{1}, j_{2}\right)}^{(32,32)}$ and (bottom) a uniform sampling of the function $h_{f}$ over the grid $\theta_{\left(j_{1}, j_{2}\right)}^{(32,32)}$. The function $f$ is defined by (5.12).

Since $f$ is a function in $L^{1}\left([-\pi, \pi]^{2}\right)$ with real Fourier coefficients, then, from Theorem 3.4, we have the following spectral distribution result:

$$
\left\{Y_{\mathbf{n}} T_{\mathbf{n}}[f]\right\}_{\mathbf{n}} \sim_{\lambda} \psi_{|f|}
$$

where $\psi_{|f|}$ is defined as in formula (3.2). Moreover, $f$ is a separable function satisfying the hypotheses of Proposition 4.1, and so also the following spectral distribution holds: 

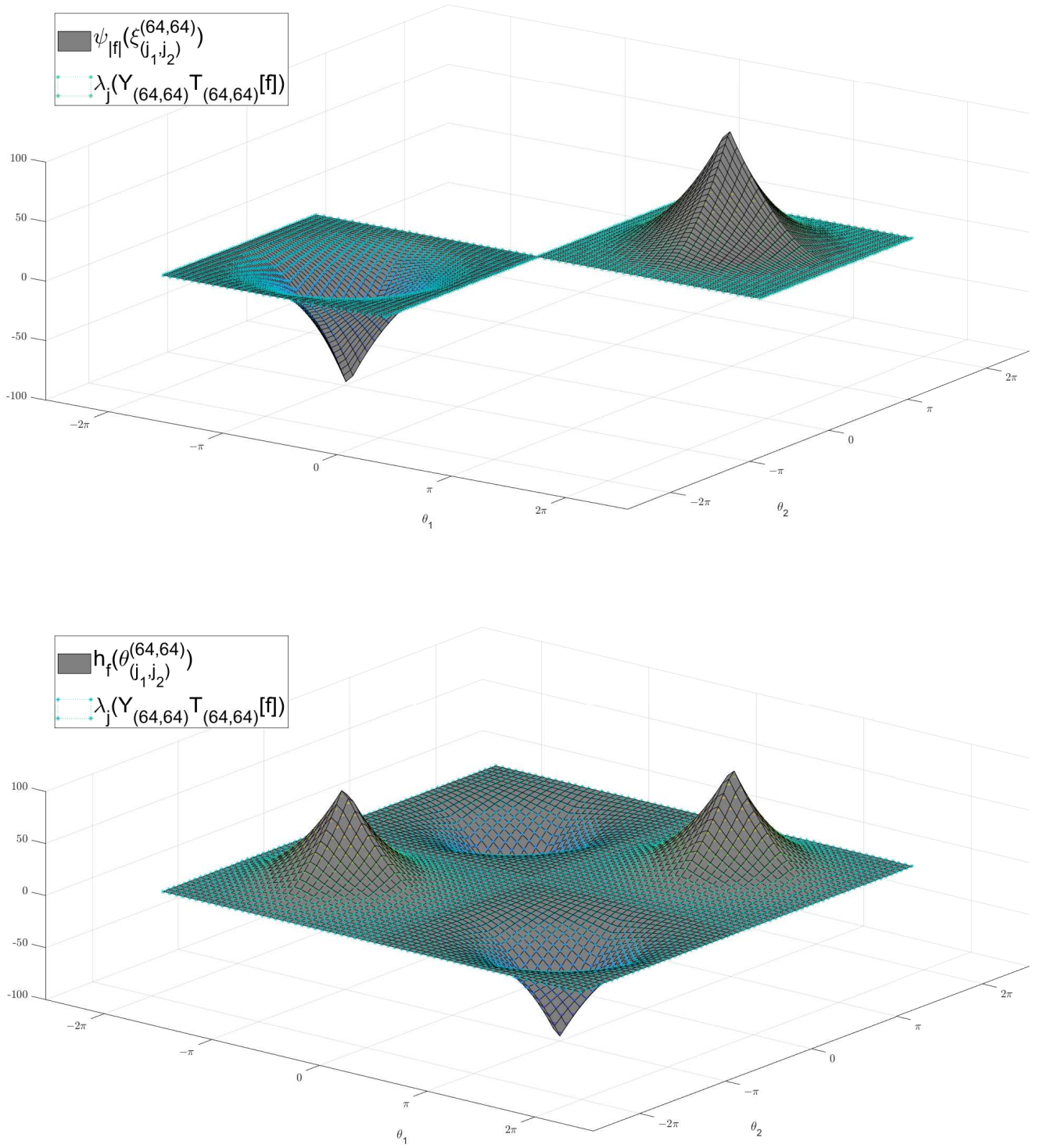

Figure 3. Example 5.3, a comparison between the eigenvalues $\lambda_{j}\left(Y_{(64,64)} T_{(64,64)}[f]\right)$ and (top) the samples $\psi_{|f|}$ over the $\operatorname{grid} \xi_{\left(j_{1}, j_{2}\right)}^{(64,64)}$ and (bottom) a uniform sampling of the function $h_{f}$ over the grid $\theta_{\left(j_{1}, j_{2}\right)}^{(64,64)}$. The function $f$ is defined by (5.15).

$$
\left\{Y_{\mathbf{n}} T_{\mathbf{n}}[f]\right\}_{\mathbf{n}} \sim_{\lambda} h_{f}
$$

where $h_{f}$ is defined as in (4.6).

In Figure 3, we numerically show that both the results are accurate in approximating the eigenvalues of $Y_{\mathbf{n}} T_{\mathbf{n}}[f]$. In particular, on the top of Figure 3, we can observe that the eigenvalues $\lambda_{j}\left(Y_{\mathbf{n}} T_{\mathbf{n}}[f]\right), j=$ $1, \ldots, N(\mathbf{n}), \mathbf{n}=(64,64)$, are well approximated by the samplings of the function $\psi_{|f|}$ over the grid $\xi_{\left(j_{1}, j_{2}\right)}^{\left(n_{1}, n_{2}\right)}$ 
defined in (5.11). Analogously, the bottom of Figure 3 numerically confirms that an accurate approximation of the eigenvalues $\lambda_{j}\left(Y_{\mathbf{n}} T_{\mathbf{n}}[f]\right), j=1, \ldots, N(\mathbf{n}), \mathbf{n}=(64,64)$, can be computed by a uniform sampling of the function $h_{f}=\psi_{\left|f_{1}\right|} \otimes \psi_{\left|f_{2}\right|}$ over the domain $[-2 \pi, 2 \pi]^{2}$.

6. Conclusions. In this note, we have proven asymptotic results concerning the singular value distribution and the spectral distribution for the considered symmetrization of multilevel Toeplitz-sequences. Taking inspiration from the unilevel matrix-sequence $\left\{Y_{n} T_{n}[f]\right\}_{n}$, we focused of the case where $f$ is a $k$ variate function in $L^{1}([-\pi, \pi])^{k}$ and $\left\{Y_{\mathbf{n}} T_{\mathbf{n}}[f]\right\}_{\mathbf{n}}$ the symmetrization of a $k$-level Toeplitz-sequence. We analyzed the case where $f$ is a trigonometric polynomial, and then we extend the result to $f \in L^{1}\left([-\pi, \pi]^{k}\right)$, $k>1$, by the notion and properties of the approximating class of sequences. In addition, we dedicated particular attention to the case where $f$ is a $k$-variate separable function. Under this additional hypothesis, the spectral distribution of the sequence $\left\{Y_{\mathbf{n}} T_{\mathbf{n}}[f]\right\}_{\mathbf{n}}$ can be obtained by a tensor product argument from the 1D setting. The study of possible simplifications when dealing with the class of polynomials will be subject of the future investigation. Moreover, as a future goal, we intend to exploit the derived knowledge of the asymptotic spectral and singular value distribution in order to design efficient solvers for large linear system stemming from practical applications and in this direction a first step is done in [13, 16].

Acknowledgment. Paola Ferrari, Isabella Furci, and Stefano Serra-Capizzano are partially supported by the INdAM Research group GNCS.

\section{REFERENCES}

[1] F. Avram. On bilinear forms in Gaussian random variables and Toeplitz matrices. Prob. Theory Related Fields, 79(1):37-45, 1988.

[2] R. Bhatia. Matrix Analysis. Springer, New York, 1997.

[3] R. Chan and X. Jin. An Introduction to Iterative Toeplitz Solvers, Vol. 5. Fundamentals of Algorithms. Society for Industrial and Applied Mathematics (SIAM), Philadelphia, PA, 2007.

[4] D. Fasino and P. Tilli. Spectral clustering properties of block multilevel Hankel matrices. Linear Algebra Appl., 306(13):155-163, 2000.

[5] P. Ferrari, I. Furci, S. Hon, M. Mursaleen, and S. Serra-Capizzano. The eigenvalue distribution of special 2-by-2 block matrix-sequences with applications to the case of symmetrized Toeplitz structures. SIAM J. Matrix Anal. Appl., 40(3):1066-1086, 2019.

[6] P. Ferrari, N. Barakitis, and S. Serra-Capizzano. Asymptotic spectra of large matrices coming from the symmetrization of Toeplitz structure functions and applications to preconditioning Numer. Linear Algebra Appl., 28:e2332, 2020.

[7] C. Garoni and S. Serra-Capizzano. Generalized Locally Toeplitz Sequences: Theory and Applications. Vol. I. Springer, Cham, 2017.

[8] C. Garoni and S. Serra-Capizzano. Generalized Locally Toeplitz Sequences: Theory and Applications. Vol. II. Springer, Cham, 2018.

[9] C. Garoni, S. Serra-Capizzano, and P. Vassalos. A general tool for determining the asymptotic spectral distribution of Hermitian matrix-sequences. Oper. Matrices, 9(3):549-561, 2015.

[10] U. Grenander and G. Szegö. Toeplitz Forms and Their Applications, second edition. Chelsea Publishing Co., New York, 1984.

[11] W. Hackbusch Iterative Solution of Large Sparse Systems of Equations. Springer-Verlag, New York, 2016.

[12] M. Mazza and J. Pestana. Spectral properties of flipped Toeplitz matrices. BIT Numer. Math., 59:463-482, 2019.

[13] M. Mazza and J. Pestana. The asymptotic spectrum of flipped multilevel Toeplitz matrices and of certain preconditionings. arXiv:2011.08372, 2020.

[14] M. Ng. Iterative Methods for Toeplitz Systems. Numerical Mathematics and Scientific Computation. Oxford University Press, New York, 2004.

[15] S. Parter. On the distribution of the singular values of Toeplitz matrices. Linear Algebra Appl., 80:115-130, 1986.

[16] J. Pestana. Preconditioners for symmetrized Toeplitz and multilevel Toeplitz matrices. SIAM J. Matrix Anal. Appl., 40(3):870-887, 2019. 
[17] J. Pestana and A. Wathen. A preconditioned MINRES method for nonsymmetric Toeplitz matrices. SIAM J. Matrix Anal. Appl., 36(1):273-288, 2015.

[18] Y. Saad Iterative Methods for Sparse Linear Systems. Society for Industrial and Applied Mathematics, Philadelphia, PA, 2003.

[19] S. Serra-Capizzano. Distribution results on the algebra generated by Toeplitz sequences: a finite-dimensional approach. Linear Algebra Appl., 328(1):121-130, 2001.

[20] S. Serra-Capizzano. Generalized locally Toeplitz sequences: spectral analysis and applications to discretized partial differential equations. Linear Algebra Appl., 366:371-402, 2003.

[21] S. Serra-Capizzano. The GLT class as a generalized Fourier analysis and applications. Linear Algebra Appl., 419(1):180233, 2006.

[22] S. Serra-Capizzano and P. Tilli. On unitarily invariant norms of matrix-valued linear positive operators. J. Inequalities Appl., 7(3):309-330, 2002.

[23] P. Tilli. A note on the spectral distribution of Toeplitz matrices. Linear and Multilinear Algebra, 45(2-3):147-159, 1998.

[24] P. Tilli. Some results on complex Toeplitz eigenvalues. J. Math. Anal. Appl., 239(2):390-401, 1999.

[25] E. Tyrtyshnikov. New theorems on the distribution of eigenvalues and singular values of multilevel Toeplitz matrices. Doklady Akademii Nauk, 333(3):300-303, 1993.

[26] E. Tyrtyshnikov. Influence of matrix operations on the distribution of eigenvalues and singular values of Toeplitz matrices. Linear Algebra Appl., 207:225-249, 1994.

[27] E. Tyrtyshnikov. A unifying approach to some old and new theorems on distribution and clustering. Linear Algebra Appl., 232:1-43, 1996.

[28] N. Zamarashkin and E. Tyrtyshnikov. Distribution of the eigenvalues and singular numbers of Toeplitz matrices under weakened requirements on the generating function. Matematicheskii Sbornik, 188(8):83-92, 1997. 